\title{
Simulación en Excel: Buscando la probabilidad de un evento
}

\author{
Giovanni Sanabria Brenes \\ gsanabriab@yahoo.com \\ Instituto Tecnológico de Costa Rica \\ Universidad de Costa Rica Costa Rica
}

\author{
Félix Núñez Vanegas. \\ fnunez@itcr.ac.cr \\ Instituto Tecnológico de Costa Rica \\ Universidad de Costa Rica Costa Rica
}

\section{Resumen.}

El presente trabajo tiene como objetivo que el lector obtenga una mejor comprensión del concepto de probabilidad y una interpretación correcta a la Ley de los Grandes Números. Las actividades planteadas adoptan el enfoque frecuencial de la definición de probabilidad, en donde a través de la simulación de algunos experimentos aleatorios utilizando Excel y desde una perspectiva Brousseauneana, se aproximan las probabilidades teóricas de algunos eventos.

Palabras clave: Didáctica, probabilidad frecuencial, ley de los grandes números, experimentación, simulación.

\section{Abstract}

This work aims the reader get a better understanding of concept of probability and a correct interpretation of the Law of Large Numbers. The proposed activities adopt the approach of frecuencial definition of probability where, through random simulation experiments using Excel and a Brousseauneana perspective, theoretical probabilities of some events are approached.

KeyWords: Teaching, Law of Large Numbers, experimentation, simulation

\subsection{Introducción}

En aquellos problemas en los que la definición clásica de probabilidad no es utilizable, como en aquellos en donde los eventos no son equiprobables o bien el número favorable de casos es desconocido o infinito, la experiencia práctica juega un papel muy importante. Así por ejemplo si queremos calcular la probabilidad de que al lanzar una moneda cargada salga escudo o corona, es útil realizar el experimento en forma práctica. No obstante, hará falta lanzar la moneda muchas veces y podría resultar tedioso y poco práctico dicho procedimiento.

Por otro lado, cuando a los estudiantes de un curso de probabilidades se les enuncia dicha definición, los ejemplos que se realizan en clases hacen pensar que el concepto quedó claro. Empero, cuando se les pregunta de si al lanzar un moneda no cargada, 10 veces, en cuántas ocasiones piensan que caerá escudo, la respuesta nos asombra. La mayoría dice que 5 veces escudo y consecuentemente 5 veces corona. Al respecto, consultar Núñez (2010). 
Lo anterior hace pensar que hay una mala interpretación del concepto de probabilidad y de la Ley de los Grandes Números.

Ante estas situaciones, se ha desarrollado una propuesta acerca del concepto de probabilidad utilizando el enfoque frecuencial o estadístico, en donde se usen los resultados obtenidos de la experiencia misma, con el fin de, por un lado, satisfacer las necesidades teóricas y prácticas de una definición de probabilidad, y por el otro, que aclare al estudiante el concepto de probabilidad teórica a través de la experiencia misma.

No obstante, como se mencionó más arriba, llevar a cabo experimentos concretos en el aula podría resultar muy tedioso y demandar mucho tiempo. Por ejemplo, lanzar una moneda 100 veces o bien 500, podría demandar mucho de la clase y tornarse muy aburrida. Es por ello que dicha propuesta brinda algunas estrategias que simulen experimentos utilizando Excel, dado que en la mayoría de las computadoras está instalado este software, lo cual permitirá concentrarse en los aspectos medulares de los conceptos.

El taller va dirigido a estudiantes universitarios pero podría adaptarse fácilmente a una población de enseñanza media, y fue planteado en principio como una propuesta didáctica en el III Encuentro de Enseñanza de la Matemática UNED, realizado en el INBio Parque, Heredia, Costa Rica, en setiembre 2010, bajo el nombre "Una propuesta para introducir el estudio de las probabilidades: Probabilidad Frecuencial" (Sanabria \& Núñez, 2010), y le hemos dado forma para llevarlo al aula como un taller con la esperanza de que los participantes adquieran los conceptos propuestos y les sean de gran utilidad, no sólo en el cálculo de algunas probabilidades y en el desarrollo de sus clases sobre estos temas, sino también, por medio de la simulación de eventos aleatorios en Excel, adquieran un concepto más sólido e intuitivo de la probabilidad así como también de la Ley de los Grandes Números.

\subsection{Objetivos}

Objetivo general. Establecer un concepto intuitivo de la probabilidad y de la Ley de los Grandes Números por medio de la simulación de eventos aleatorios en Excel.

\section{Objetivos Específicos.}

- Describir los conceptos de espacio muestral, evento, eventualidad, tipos de eventos y eventos compuestos

- Aplicar correctamente la Ley de los Grandes Números

- Simular eventos aleatorios en Excel

- Resolver problemas que involucran el cálculo de probabilidades por medio de la simulación utilizando Excel

- Resolver el problema de Monty Hall por medio de la simulación utilizando Excel

Para el desarrollo del trabajo se requiere una computadora con Excel 2007 en español

\subsection{Fundamentos teóricos}

Fundamento matemático. Dado un experimento probabilístico, sea $\Omega$ el espacio muestral y A un evento. Si el experimento se repite $n$ veces, se define $X(n)$ como el número de veces que ocurre $A$ de las $n$. La Ley de los grandes números, establece que para $n$ grande, se cumple que:

Simulación en Excel: Buscando la probabilidad de un evento. G. Sanabria B.,F. Núñez V.

Derechos Reservados (C) 2012 Revista digital Matemática, Educación e Internet (www.tec-digital.itcr.ac.cr/revistamatematica/) 


$$
P(A) \approx \frac{X(n)}{n}
$$

Formalmente este resultado se describe en el siguiente teorema.

Teorema 1.1 (Ley de los Grandes Números). Dado un experimento, sea $A$ un evento y $X(n)$ el número de veces que ocurre $A$ en $n$ de estos experimentos, entonces para todo $\varepsilon>0$ se tiene que

$$
P\left(\left|\frac{X(n)}{n}-P(A)\right| \geqslant \varepsilon\right)=0
$$

para $n$ suficientemente grande.

Es decir, existe un $n$ a partir del cual la probabilidad de que la diferencia entre $X(n) / n$ y $P(A)$ sea mayor a $\varepsilon$ es cero. La idea es, en primera instancia, identificar el valor de $X(n) / n$ como la probabilidad frecuencial del evento A. Así, la Ley de los Grandes Números establece condiciones para que la probabilidad frecuencial se acerque a la probabilidad teórica. La probabilidad frecuencial permite introducir la simulación de experimentos aleatorios y es rica en intuición.

Fundamento pedagógico. Basado en una perspectiva constructivista, Brousseau (1986) abordó el problema de la didáctica de la matemática a través de la teoría de situaciones. Para él, el profesor debe diseñar situaciones didácticas (problemas) en la que la solución encontrada sea el conocimiento que se desea establecer. Se debe plantear el problema al estudiante y lograr que se dé la devolución, es decir, que el estudiante se interese en el problema y donde se le devuelve la responsabilidad de su propio aprendizaje. Esto se hace en situación adidáctica, puesto que al estudiante se le pone a distancia con la intención de enseñarle algún conocimiento. En esta etapa se dan situaciones de formulación y validación, en la que el estudiante ensaya, falla, corrige y se supera. Una vez que ha resuelto el problema, el profesor en la etapa de institucionalización, enuncia el resultado obtenido por el estudiante.

Inspirada en esa concepción brousseauneana, hemos desarrollado este taller. En ese sentido se diseñaron una serie de situaciones problema que tienen la finalidad de que al resolverlas, el participante obtenga un conocimiento particular tanto del concepto de la probabilidad como el de la Ley de los Grandes Números. Luego este conocimiento será institucionalizado por los profesores encargados del taller, y finalmente se proponen situaciones problema con el fin de profundizar los saberes adquiridos.

Por otro lado, el uso de la tecnología en la etapa de formulación podría ser muy importante en la solución del problema probabilístico planteado, específicamente, el uso de la computadora. Al adoptar la definición clásica de probabilidad, se presenta el problema de saber cuál es el número de casos favorables, y nos enfrentamos entonces a los problemas de conteo, que por demás está decirlo son difíciles. De acuerdo con Antibí citado por Núñez (2005) la solución tradicional a este tipo de problemas se hace de manera escueta y carece del rigor con que se resuelven problemas de otros dominios de la matemática, lo cual dificulta controlar la solución dada. Por lo general no se está seguro de si el cálculo realizado está correcto. Por otro lado, Sanabria (2010) brinda una propuesta de cómo abordar los problemas de conteo, justamente por considerarlos difíciles. No obstante, estas estrategias están dirigidas a una población universitaria con ciertas competencias. Cuando se utiliza el enfoque frecuencial, al menos se está seguro de los casos totales, puesto que provienen de la experiencia misma, y de esta forma se tendría una buena aproximación de la probabilidad teórica de un determinado evento y a su vez se podrían confrontar los resultados de un conteo con la probabilidad frecuencial hallada en ese mismo problema.

La simulación viene a ayudar a acercarse al valor de dicha probabilidad. Cuando la probabilidad frecuencial de un evento se calcula con una muestra de tamaño n, se tendrá un valor distinto al que se obtendría con otras 
muestras del mismo tamaño, habrá algunas fluctuaciones, aunque se concentrarán alrededor de un valor específico. Es sabido que dichas diferencias o cambios en dichas probabilidades se pueden reducir si el tamaño de la muestra es considerablemente grande. Es importante que el estudiante descubra este hecho por sí mismo, a través de un descubrimiento en la solución de un problema propuesto. Es por ello que la simulación de un experimento aleatorio, utilizando un software, viene a significar una gran ayuda en la comprensión del concepto de probabilidad.

\subsection{Propuesta}

Funciones en Excel. Se pretende utilizar un número muy reducido de herramientas en Excel, que corresponden a las siguientes:

- ALEATORIO.ENTRE (min; max), la cual devuelve un número entero aleatorio entre los enterosmin y max

- CONTAR.SI (Rango; "Texto") . Esta función calcula las veces que aparece Texto en el rango

- SI (cond;res1;res2). Devuelve el res1 si se cumple la condición cond, de lo contrario devuelve el resultado res2.

- $A B S(x)$. Devuelve el valor absoluto de $x$.

Para familiarizarse con dichas funciones, realice los siguientes ejercicios:

Ejercicio 1. Desarrolle una lista de 100 números aleatorios del 1 al 10. ¿Cuántos son mayores a 7 ?

Ejercicio 2. Se tienen tres puertas, enumeradas del 1 al 3. Considere el experimento en el cual: Juan abre una puerta al azar. Luego Ana abre una puerta al azar que no sea la que abrió Juan. Simule el experimento 100 veces. ¿Cuántas veces Ana abre la puerta 1?

Ejercicio 3. Se tienen tres películas, enumeradas del 1 al 3. Considere el experimento en el cual: Karla elige una película al azar, Anthony elige otra película al azar (puede ser la misma que la de Karla) y Jorge elige una película distinta a las elegidas por Anthony, Karla y Juan. Simule el experimento 500 veces. ¿Cuántas veces elige Jorge la película 1?

Conceptos Básicos. Se describen brevemente y ejemplifican los principales conceptos básicos de probabilidad.

Los conceptos utilizados son:

- Espacio muestral: Es el conjunto de todos los posibles resultados, este se denota: $\Omega$

- Eventualidad: Es un resultado particular, es decir un elemento de $\Omega$.

- Evento: Es un conjunto de resultados, es decir un subconjunto de $\Omega$.

- Ocurrencia de un evento: Se dice que un evento ocurre si sucede una y solo una de sus eventualidades.

- Evento casi seguro: $\Omega$

- Evento casi imposible: $\varphi$ 
Ejemplo 1.1 Considere el experimento "Tirar un dado". El espacio muestral es:

$\Omega=\{1,2,3,4,5,6\}$. Observe que 6 es una eventualidad. Algunos eventos son:

A: el resultado del dado es impar

$B$ : el resultado del dado es mayor a 4

Note que $A=-1,3,5^{\prime \prime} \subseteq \Omega, B=-5,6^{\prime \prime} \subseteq \Omega$. Si el resultado del dado es 3 entonces se dice que el evento $A$ ocurre, el Evento $B$ no ocurre.

Teorema 1.2 (Eventos Compuestos). Si $A$ y $B$ son eventos entonces: $A \cup B, A \cap B, A-B$ y $A \triangle B$ son eventos.

Ejercicio 4. Se tiene una canasta con 15 bolas enumeradas del uno al quince. Las bolas con número del 1 al 7 son rojas y las demás son verdes. Considere el experimento que consiste en elegir una bola al azar de la canasta. Dados los eventos:

A: la bola elegida es verde

$B$ : la bola elegida es roja

C: la bola elegida tiene un número par

Describa la ocurrencia de los eventos $B \cup C, A \cap C, C-A$ y $C \triangle B$.

Acercamiento al concepto de probabilidad. Dado un experimento, la probabilidad o medida de posibilidad de que ocurra un evento determinado $A$ será un número entre 0 y 1 , que se interpreta como un porcentaje. Así si la probabilidad de $A$ es 0.8 , esto indica que el evento tiene un $80 \%$ de posibilidad de ocurrir.

¿Cómo determinar intuitivamente la probabilidad de que ocurra un evento? Para que la probabilidad sea útil debe existir una correspondencia entre la probabilidad y la realidad, es decir si el experimento se repite varias veces, la frecuencia relativa observada con que ocurre un evento debe ser cercana a la medida de la posibilidad de que ocurra ese evento. $A$ esta frecuencia relativa observada se le llamará probabilidad frecuencial, la cual se espera que, bajo ciertas condiciones, se aproxime a la probabilidad de que ocurra el evento (llamada probabilidad teórica)

Ejemplo 1.2 Dado el fenómeno de lanzar un dado, ¿Cuál es la probabilidad de que salga un 6 ?.

Se lanza un dado 100 veces y se observa que en 15 veces se obtiene un 6, por lo tanto la probabilidad frecuencial observada de obtener un 6 es 15/100 =15\%. La probabilidad teórica de dicho evento es $(1 / 6)=16.6 \%$ Ambos resultados son muy parecidos.

Ejercicio 5. Aproxime la probabilidad de que:

- Al lanzar dos monedas se obtengan dos caras

- Un número de 4 dígitos elegidos al azar la suma de sus dígitos sea divisible por 6 
¿Funciona o no la probabilidad frecuencial?. Se discuten en parejas los siguientes problemas:

Ejemplo 1.3 (¿Juegas o no?). En las fiestas cívicas de Zapote hay un puesto donde por 1000 colones se puede jugar DADOS A SEIS. Este juego consiste en lanzar dos dados distintos, si la suma de los resultados de los dados es menor o igual a 6 se gana el juego si no se pierde. Karla, Jorge y Anthony desean determinar si vale la pena jugar el juego. Para ello deciden que cada uno juegue veinte veces DADOS A SEIS obteniendo los siguientes resultados:

\begin{tabular}{|l|l|l|l|}
\hline & \# de veces que se ganó & Probabilidad frecuencial de ganar & ¿Vale la pena Jugar? \\
\hline Karla & 7 & $7 / 20=5 \%$ & NO \\
\hline Jorge & 10 & $10 / 20=50 \%$ & Es indiferente \\
\hline Anthony & 12 & $12 / 20=60 \%$ & SI \\
\hline
\end{tabular}

Se puede apreciar que los resultados obtenidos utilizando la probabilidad frecuencial son muy distintos. Tal parece que algunas probabilidades frecuenciales no se acercan al valor real de la probabilidad. ¿Cuál es realmente la probabilidad de ganar DADOS A SEIS?

Ejemplo 1.4 (¡El falso determinismo!). Un software asegura que detecta el $90 \%$ de los fraudes bancarios que ocurren en las tarjetas. Ante esto el Banco de Los Sueños decide adquirir el software para detectar los fraudes que les ocurren a sus clientes en las tarjetas. Sin embargo, en el primer momento de uso, el software no detectó un fraude. El banco decide demandar a la empresa, pero al revisar el software, resulta que los cálculos están bien hechos. ¿Qué está sucediendo entonces?

Los dos últimos ejemplos revelan que no necesariamente la probabilidad frecuencial se va a acercar a la probabilidad real. Entonces ¿qué condiciones deben cumplirse para que la frecuencia relativa observada se acerque a la probabilidad teórica?

La Ley de los Grandes Números. Considere un experimento, sea $A$ un evento. Esta ley indica que si el experimento se repite un número suficientemente grande de veces, entonces la probabilidad frecuencial de $A$ será muy cercana al valor real de la probabilidad. Donde el número de veces que se repite el experimento depende de la variabilidad de sus resultados.

Ejemplo 1.5 (Verificando la Ley de los grandes números). Al lanzar una moneda legal se sabe que hay un 50\% deprobabilidad de que salga escudo.

Se desea simular esta situación utilizando Excel. Para ello utilicemos la función ALEATORIO.ENTRE (min; max) la cual, como habíamos visto más arriba, devuelve un número entero aleatorio entre los enteros min y max. Así en la celda A1 de Excel se copia ALEATORIO.ENTRE (min; max), como se muestra en la figura a la derecha.

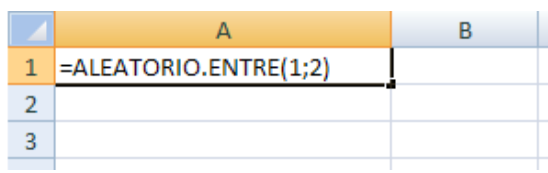


Se va a considerar el 1 como CORONA y el 2 como ESCUDO. Luego, utilizando el mouse se puede arrastrar esta fórmula hasta la celda A10 obteniendo por ejemplo, los valores que se muestran en la figura a la derecha.

Aquí se están simulando 10 lanzamientos de una moneda. En este caso la probabi-lidad frecuencial de obtener un ESCUDO es $2 / 10=20 \%$ que es muy alejada de la probabilidad esperada de $50 \%$. Para lanzar nuevamente la moneda, basta actualizar las celdas, esto se logra escribiendo algo (por ejemplo un igual o dar suprimir) en una celda desocupada. Sin embargo, al cambiar nuevamente los valores, se obtiene que en la mayoría de los casos, la probabilidad frecuencial se aleja de la probabilidad real de 50\%. Esto se debe a que el número de lanzamientos es insuficiente.

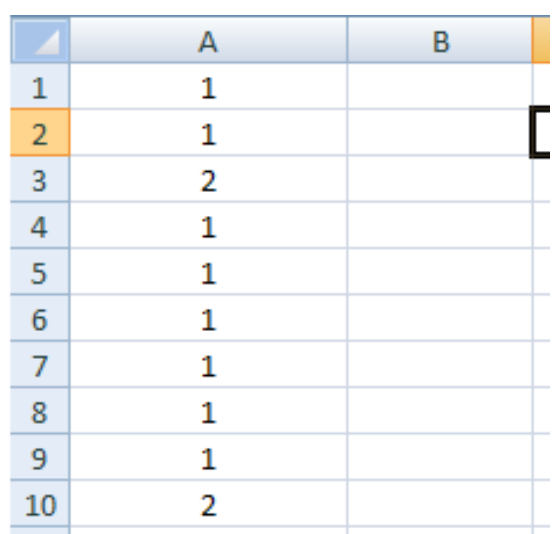

¿Qué sucede si se realizan varios lanzamientos? Suponga que se tiene diez personas y cada una simula diez lanzamientos como se vio anteriormente y obtienen los siguientes resultados:

\begin{tabular}{|c|c|c|c|c|c|c|c|c|c|c|}
\hline & \multicolumn{10}{|c|}{ Persona } \\
\hline$\#$ & $a$ & $\mathrm{~b}$ & c & $\mathrm{d}$ & $\mathrm{e}$ & $f$ & $\mathrm{~g}$ & $\mathrm{~h}$ & $i$ & j \\
\hline 1 & 1 & 2 & 2 & 2 & 2 & 2 & 1 & 2 & 2 & 2 \\
\hline 2 & 1 & 2 & 1 & 2 & 1 & 2 & 1 & 2 & 1 & 2 \\
\hline 3 & 1 & 2 & 1 & 2 & 2 & 2 & 1 & 1 & 1 & 2 \\
\hline 4 & 1 & 2 & 1 & 2 & 2 & 2 & 2 & 1 & 2 & 1 \\
\hline 5 & 2 & 1 & 1 & 2 & 1 & 2 & 2 & 1 & 2 & 2 \\
\hline 6 & 2 & 1 & 1 & 1 & 2 & 1 & 2 & 2 & 1 & 1 \\
\hline 7 & 2 & 1 & 2 & 2 & 2 & 2 & 2 & 1 & 1 & 1 \\
\hline 8 & 1 & 2 & 1 & 1 & 1 & 2 & 2 & 2 & 2 & 2 \\
\hline$\overline{9}$ & 1 & 2 & 1 & 2 & 1 & 2 & 2 & 1 & 1 & 1 \\
\hline 10 & 1 & 2 & 1 & 1 & 2 & 1 & 2 & 1 & 2 & 2 \\
\hline & & & & & & & & & & \\
\hline $\begin{array}{l}\text { probabilidad } \\
\text { frecuencial }\end{array}$ & 0,3 & 0,7 & 0,2 & 0,7 & 0,6 & 0,8 & 0,7 & 0,4 & 0,5 & 0,6 \\
\hline
\end{tabular}

Observe que la mayoría de probabilidades frecuenciales obtenidas por cada persona distan de la esperada. Sin embargo, si se consideran todos los lanzamientos, entonces la probabilidad frecuencial de obtener ESCUDO es $((55) /(100))=55 \%$ que es bastante cercana al $50 \%$.

Ejemplo 1.6 (¿Juegas o no?). Las distintas respuestas a la pregunta ¿Vale la pena Jugar DADOS A SEIS? del ejemplo (3) se debe a las pocas veces que se jugó el juego. Simulemos este juego 100 veces. Para ello se escribe en la hoja de Excel:

\begin{tabular}{|l|l|l|l|}
\hline Celda: & A1 & B1 & C1 \\
\hline Escribir: & Dado1 & Dado2 & ¿Ganó? \\
\hline Celda: & A2 & B2 & C2 \\
\hline Escribir: & =ALEATORIO.ENTRE $(1 ; 6)$ & =ALEATORIO.ENTRE $(1 ; 6)$ & $=$ SI (A2+B2<=6; "SI" ; "NO") \\
\hline
\end{tabular}

Recordemos que la función SI (cond; res1; res2) devuelve el res1 si se cumple la condición cond, de lo contrario devuelve el resultado res2. Note que si la suma de los resultados de los dados es menor a $6(\mathrm{~A} 2+\mathrm{B} 2<=6$ ) entonces se da con respuesta SI, esto por cuanto sí se ganó el juego. Hasta el momento se ha simulado sólo un juego, donde 
un posible resultado del mismo es:

\begin{tabular}{|l|c|c|c|c}
\hline \hline & A & B & C & \\
\hline 1 & Dado 1 & Dado2 & ¿Ganó? & \\
\hline 2 & 4 & 1 & SI & \\
\hline 3 & & & \\
\hline
\end{tabular}

Para simular 100 juegos basta seleccionar las celdas escritas de la fila 2 y con el mouse arrastrar estas fórmulas hasta la fila 101, obteniendo

\begin{tabular}{|c|c|c|c|c|}
\hline & A & B & C \\
\hline 1 & Dado 1 & Dado2 & ¿Ganó? \\
\hline 2 & 3 & 4 & NO \\
\hline 3 & 6 & 6 & NO \\
\hline 4 & $\vdots$ & $\vdots$ & $\vdots$ \\
\hline 99 & 5 & 6 & NO \\
\hline 100 & 1 & 1 & SI \\
\hline 101 & 2 & 1 & SI \\
\hline 102 & & & \\
\hline
\end{tabular}

Para determinar cuántas veces se ganó el juego de las 100 partidas se puede escribir en una celda vacía

$$
=\text { CONTAR.SI }(\text { C2:C101;"=SI") }
$$

La función CONTAR. S I calcula las veces que aparece SI en las celdas respectivas de la columna C. En nuestro caso, el valor que da esta calda es 44 . Por lo tanto, la probabilidad frecuencial de ganar el juego es de $44 \%$. La probabilidad teórica de ganar el juego es de $5 / 12 \approx 41.67 \%$. Así no vale la pena jugar DADOS A SEIS.

En el ejemplo anterior la probabilidad frecuencial no está lo suficientemente cerca de la probabilidad teórica. Esto se debe a que, a diferencia del ejercicio tras anterior, hay mayor variabilidad en los resultados del experimento. Es decir, lanzar un dado tiene más resultados que lanzar una moneda, por lo que se requiere un número mayor de repeticiones del experimento para que la probabilidad frecuencial este suficientemente cercana a la probabilidad real.

Ejemplo 1.7 (¡El falso determinismo!). La Ley de los Grandes nos ayuda a justificar el falso determinismo indicado en el ejemplo (4). El hecho de que exista una probabilidad alta (90\%) de que el software detecte fraudes no significa que se puede asegurar que va a detectar el primer fraude o los primeros fraudes, sino que en un número suficientemente grande de fraudes, detectará alrededor del 90\%. Simulemos, en Excel esto en un número pequeño de fraudes (diez). Para ello escriba en las respectivas celdas:

\begin{tabular}{|l|l|l|}
\hline Celda: & A1 & B1 \\
\hline Escribir: & $=$ ALEATORIO () & SI $($ A $1<=0.9 ;$ "Se detectó" ; "No se detectó") \\
\hline
\end{tabular}

La Función ALEATORIO () indica un número real aleatorio entre 0 y 1 . Esto nos permite simular los resultados del software, si el valor dado por ALEATORIO() es menor igual a 0.9 el software detectó el fraude, de lo contrario no lo detecta. Luego, como hemos visto, con el mouse se arrastran estas fórmulas hasta la fila 10. Si bien se puede obtener una simulación donde prácticamente los diez fraudes fueron detectados, fácilmente (actualizando las celdas) se obtienen casos como este: 


\begin{tabular}{|r|r|r|}
\hline 1 & 0,68750046 & Se detectó \\
\hline 2 & 0,97619271 & No se detectó \\
\hline 3 & 0,26931474 & Se detectó \\
\hline 4 & 0,16517495 & Se detectó \\
\hline 5 & 0,73325355 & Se detectó \\
\hline 6 & 0,94599857 & No se detectó \\
\hline 7 & 0,20288272 & Se detectó \\
\hline 8 & 0,66723848 & Se detectó \\
\hline 9 & 0,98883887 & No se detectó \\
\hline 10 & 0,99951653 & No se detectó \\
\hline.. & & \\
\hline
\end{tabular}

Aquí la probabilidad frecuencial de detectar fraudes es de $60 \%$ muy alejada del $90 \%$, pero el software, bajo nuestra simulación está trabajando bien.

Ejercicio 6. Suponga que hay una alta probabilidad de ganar un juego de apuestas, ¿significa esto que en las primeras partidas se gana? Si un jugador tiene una o algunas derrotas iniciales, ¿debe retirarse? Pero, ¿qué sucede si sigue jugando un número suficiente de veces?

Ejercicio 7 Suponga que hay una baja probabilidad de ganar un juego de apuestas con un premio de un millón de dólares ¿Se puede asegurar que si apuesta entonces perderá? Si un jugador gana el juego, ¿debe retirarse? , ¿qué sucede si sigue jugando un número suficiente de veces?

Ejercicio 8. (La ley de los grandes números: Valores absolutos o relativos). Explore con Excel la siguiente afirmación: De acuerdo con la Ley de los Grandes Números, entre más veces se tira una moneda, más cerca se estará el número obtenido de escudos de la mitad del total de los lanzamientos.

La afirmación del ejercicio anterior es falsa. La Ley de los grandes Números se refiere a valores relativos no absolutos. Dicha afirmación es una malinterpretación frecuente de la Ley de los grandes números y se desmiente en el siguiente ejemplo.

Ejemplo 1.8 En la siguiente tabla se describen los resultados en dos secuencias de lanzamientos de una moneda legal:

\begin{tabular}{|l|l|l|l|}
\hline \# de lanzamientos & \# de escudos & $\begin{array}{l}\text { Diferencia entre el \# de escu- } \\
\text { dos y la mitad de los lanza- } \\
\text { mientos }\end{array}$ & $\begin{array}{l}\text { Probabilidad frecuencial de } \\
\text { obtener un escudo }\end{array}$ \\
\hline 20 & 8 & 2 & $8 / 20=40 \%$ \\
\hline 200 & 95 & 5 & $95 / 200=47.5 \%$ \\
\hline
\end{tabular}

Como se aprecia en este caso, al aumentar el número de lanzamiento, pese a que en términos absolutos el número de escudos se aleja de la mitad de lanzamientos, en términos relativos se acerca al 50\% de los lanzamientos. Esto se debe a que una diferencia de una unidad entre el número de escudos y la mitad de lanzamientos no pesa igual en 20 lanzamientos que en 200 lanzamientos. 


\subsection{Resolución de algunos problemas}

Ejemplo 1.9 (La bola de fútbol). En un refresco que compró Juan en la pulpería la MINITA, cercana a su colegio, se ganó una bola de fútbol. Sin embargo, al reclamar su premio en la MINITA, la encargada le indicó que el premio solamente se lo puede dar el camión repartidor y únicamente pasa el Martes entre 10am y 11am aleatoriamente, y en la pulpería se queda exactamente 10 minutos. Dado que Juan está en clases ese día, decide elegir al azar un tiempo entre 10am y $11 \mathrm{pm}$ para fugarse de clases y esperar en la pulpería exactamente diez minutos para ver si logra encontrarse con el camión repartidor. ¿Cuál es la probabilidad de que el martes obtenga su premio?

Utilizamos Excel para modelar el problema. Para ello escriba en las respectivas celdas:

\begin{tabular}{|l|l|l|l|}
\hline Celda: & A1 & B1 & C1 \\
\hline Escribir: & $\begin{array}{l}\text { Hora de llegada del } \\
\text { camión }\end{array}$ & $\begin{array}{l}\text { Hora de llegada de } \\
\text { Juan }\end{array}$ & ¿Obtiene el premio? \\
\hline Celda: & A2 & B2 & C2 \\
\hline Escribir: & $=$ ALEATORIO () & $=$ ALEATORIO () & $=$ SI (ABS (A2-B2) <=1/6; "SI" ; "NO" ) \\
\hline
\end{tabular}

Se interpretará el valor de ALEATORIO() como los minutos en horas después de las 10am. Así., si en la celda A2 el valor de ALEATORIO() es 0,15 se tiene que $0,15 \mathrm{~h}=9 \mathrm{~min}$, por lo tanto la hora de llegada del camión sería a las 10:09 am. Por otro lado, la función ABS (num) devuelve el valor absoluto de num. Así, dado que ambos (Juan y el camión) esperan 10 minutos que equivale a (1/6) de hora, si la diferencia entre las horas de llegada es menor a (1/6) , entonces Juan obtiene el premio ese martes, de lo contrario debe esperar al siguiente. Para simular esta situación 500 veces, como se ha visto, se seleccionan las celdas escritas de la fila 2 y con el mouse se arrastran estas fórmulas hasta la fila 501, obteniendo:

\begin{tabular}{|c|c|c|c|}
\hline & A & B & C \\
\hline 1 & Hora de llegada del camión & Hora de llegada de Juan & ¿Obtiene el premio? \\
\hline 2 & 0,696406965 & 0,872092338 & NO \\
\hline 3 & 0,598863964 & 0,667165174 & SI \\
\hline 4 & 0,044151278 & 0,270634535 & NO \\
\hline 5 & $\vdots$ & $\vdots$ & $\vdots$ \\
\hline 499 & 0,885655151 & 0,836425422 & SI \\
\hline 500 & 0,919716788 & 0,835197463 & SI \\
\hline 501 & 0,205350839 & 0,578373853 & NO \\
\hline.-+ & & & \\
\hline
\end{tabular}

Por ejemplo, en la tercer simulación se tiene que

$$
0.598863964 \mathrm{~h} \approx 35.93 \mathrm{~min} \text { y } 0.667164174 \mathrm{~h} \approx 40.03 \mathrm{~min}
$$

Por lo tanto el camión llega aproximadamente a las 10:36 am y Juan a las 10:40 am, por lo tanto Juan obtiene su premio. De las 500 simulaciones, el número de veces que Juan logra obtener su premio se obtiene escribiendo en una celda vacía:

$$
=\text { CONTAR.SI (C2:C501;"=SI") }
$$

Simulación en Excel: Buscando la probabilidad de un evento. G. Sanabria B.,F. Núñez V.

Derechos Reservados (C) 2012 Revista digital Matemática, Educación e Internet (www.tec-digital.itcr.ac.cr/revistamatematica/) 
En nuestro caso, este valor es de 157. Por lo tanto, la probabilidad frecuencial de que Juan obtenga el próximo martes la bola es de $157 / 500=31.4 \%$. La probabilidad real es de $11 / 36 \approx 30.56 \%$.

Ejemplo 1.10 (El Problema de Monty Hall). En un concurso se tienen tres puertas, detrás de una de ellas hay un auto y detrás de las otras hay una cabra. El participante debe elegir una de las tres puertas, sin abrirla. Después Monty, el presentador, abre una de las dos puertas restantes en la que hay una cabra. Así quedan dos puertas sin abrir una con auto y otra con cabra. Monty ofrece la posibilidad al concursante de cambiar su puerta o permanecer con su elección. ¿Qué es mejor, cambiar de puerta o no? (Este problema es uno de los más controversiales en probabilidad y se basa en un programa de televisión de los 70's).

En primera instancia se puede creer, como muchos Matemáticos en el pasado, que pasarse de puerta no influye en la probabilidad de ganar el auto. Sin embargo, al simular el problema podemos obtener sorpresas. Primero, la pregunta a responder es equivalente a la siguiente: Si el participante decide cambiarse de puerta, ¿cuál es la probabilidad de ganar el auto? Utilizando Excel para modelar el problema, suponga que las puertas están numeradas del 1 al 3. Primero debemos elegir la puerta donde está el premio y la puerta elegida inicialmente por el participante, para ello escribamos en las respectivas celdas:

\begin{tabular}{|l|l|l|}
\hline Celda: & A1 & B1 \\
\hline Escribir: & Puerta donde está el auto & Puerta elegida inicialmente \\
\hline Celda: & A2 & B2 \\
\hline Escribir: & =ALEATORIO.ENTRE $(1 ; 3)$ & =ALEATORIO.ENTRE $(1 ; 3)$ \\
\hline
\end{tabular}

Luego en la celda C1 se escribe: Puerta abierta por el presentador. ¿Qué puerta abre Monthy? Si la puerta donde se encuentra el auto y la elegida inicialmente son distintas, entonces Monty abre la única distinta a éstas: tal puerta es la número 6-A2-B2, esto por cuanto las puertas están enumeradas con 1, 2 y 3 . Al sumar dichas numeraciones, obtenemos 6. Así que si el concursante eligió la puerta 2 y el auto se encuentra en la puerta 3, Monty abrirá la número $6-2-3=1$, Pero, si las puerta A2 y B2 son la misma, entonces Monty elige la puerta que va a abrir al azar. Así se escribe en la celda $\mathrm{C} 2$ :

$$
\begin{gathered}
=\mathrm{SI}(\mathrm{NO}(\mathrm{B} 2=\mathrm{A} 2) ; 6-\mathrm{A} 2-\mathrm{B} 2 ; \mathrm{SI}(\mathrm{NO}(\mathrm{B} 2=1) ; \\
\mathrm{SI}(\mathrm{ALEATORIO}()<0,5 ; 1 ; 6-\mathrm{A} 2-1) ; \mathrm{SI}(\operatorname{ALEATORIO}()<0,5 ; 2 ; 3)))
\end{gathered}
$$

\begin{tabular}{|c|c|c|c|}
\hline Celda: & D1 & E1 & F1 \\
\hline Escribir: & ¿Se pasa de puerta? & $\begin{array}{l}\text { Puerta elegida al fi- } \\
\text { nal }\end{array}$ & ¿Ganó el auto? \\
\hline Celda: & D2 & E2 & F2 \\
\hline Escribir: & $\begin{array}{l}=\text { SI (ALEATORIO ( ) <0, } 5 \\
; " \text { "SI"; "NO") }\end{array}$ & $\begin{array}{l}=\mathrm{SI}(\mathrm{D} 2=" \mathrm{SI} " ; \\
6-\mathrm{C} 2-\mathrm{B} 2 ; \mathrm{B} 2)\end{array}$ & $\begin{array}{l}=\text { SI (Y (D2="SI";E2=A2); "pasó y ganó"; } \\
\text { SI (Y (D2="NO";E2=A2); "no pasó y } \\
\text { ganó";"perdió")) }\end{array}$ \\
\hline
\end{tabular}

Posteriormente se escribe las celdas respectivas:

¿Se pasa de puerta? Dado que Monty abrió una puerta, entonces dado que las 2 puertas tienen la misma probabilidad de ser escogida, hay un $50 \%$ de probabilidad de pasarse de puerta. La puerta elegida al final depende de la decisión tomada en D2. Luego, se enuncian los diferentes resultados en F2, se diferencia si se ganó el auto por cambiarse o no de puerta. Para simular esta concurso 2000 veces, como se ha visto, se seleccionan las celdas escritas 
de la fila 2 y con el mouse se arrastran estas fórmulas hasta la fila 2001, obteniendo por ejemplo:

\begin{tabular}{|c|c|c|c|c|c|c|}
\hline & A & B & C & D & E & F \\
\hline 1 & $\begin{array}{c}\text { Puerta } \\
\text { donde está } \\
\text { el auto }\end{array}$ & $\begin{array}{c}\text { Puerta } \\
\text { elegida } \\
\text { inicialmente }\end{array}$ & $\begin{array}{c}\text { Puerta abierta } \\
\text { por el } \\
\text { presentador }\end{array}$ & $\begin{array}{c}\text { ¿Se pasa } \\
\text { de } \\
\text { puerta? }\end{array}$ & $\begin{array}{c}\text { Puerta } \\
\text { elegida } \\
\text { al final }\end{array}$ & $\begin{array}{c}\text { ¿Ganó el } \\
\text { auto? }\end{array}$ \\
\hline 2 & 2 & 1 & 3 & SI & 2 & paso y ganó \\
\hline 3 & 1 & 2 & 3 & NO & 2 & perdió \\
\hline 4 & 1 & 2 & 3 & SI & 1 & paso y ganó \\
\hline 5 & 2 & 3 & 1 & SI & 2 & paso y ganó \\
\hline 6 & $\vdots$ & $\vdots$ & $\vdots$ & $\vdots$ & $\vdots$ & $\vdots$ \\
\hline 1999 & 3 & 1 & 2 & NO & 1 & perdió \\
\hline 2000 & 2 & 2 & 3 & NO & 2 & no paso y ganó \\
\hline 2001 & 1 & 1 & 2 & NO & 1 & no paso y ganó \\
\hline $7 n n$ & & & & & & \\
\hline
\end{tabular}

Si el participante decide cambiarse de puerta, la probabilidad frecuencial de ganar el auto está dado por

(\# de veces que se pasó y ganó)/(\# de veces que se pasa de puerta)

Para obtener este valor, se escribe en una celda vacía

=CONTAR.SI (F2:F2001; "paso y ganó") /CONTAR.SI (D2:D2001;"SI")

En nuestro caso, la probabilidad frecuencial es aproximadamente de $67,92 \%$ que es cercana a la probabilidad real de cambiarse de puerta, la cual es de $2 / 3$.

Ejercicio 9. (Los fantasmas). En una noche de este mes 4 fantasmas de Tibás salen a asustar por la noche a San José. En su trabajo se encuentran con dos amigos fantasmas provenientes de Guanacaste. Por lo emocionante de su labor los sorprende el amanecer y se meten a las 4 cuevas de los fantasmas de Tibás ocupándolas de manera aleatoria. ¿Cuál es la probabilidad de que al distribuirse en las cuevas ninguna cueva quede desocupada?

Ejercicio 10. (La aguja de Buffon). Se tiene una tabla marcada por infinitas líneas paralelas donde la distancia entre dos líneas consecutivas es $A=6 \mathrm{~cm}$. Una aguja de largo $L=4 \mathrm{~cm}$ es lanzada al azar a la tabla.

1. ¿Cuál es la probabilidad $P$ de que la aguja toque una de las líneas paralelas? Sugerencia: Sea $C$ el punto medio de la aguja, considere las variables $X$ : la distancia de $C$ a la recta paralela más cercana, $\alpha$ : el ángulo no obtuso formado por la aguja y la proyección perpendicular de $C$ a la recta paralela más cercana. Los valores de $X$ y $\alpha$ son valores aleatorios entre determinados rangos. ¿Qué condición deben cumplir los valores de $X$ y $\alpha$ para que la aguja toque las líneas?

2. Considere el valor $Y=(2 L) /(P A)$. ¿Cómo se pueden determinar aproximaciones cada vez mejores del valor de $Y$ ? Determine algunas de estas aproximaciones y deduzca el valor de $Y$.

\subsection{Conclusión}

A través del enfoque frecuencial, el abordaje del concepto de probabilidad resulta más natural y adecuado. A través de las simulaciones hechas en Excel, se logran realizar una cantidad considerable de repeticiones de un experimento 
con muestras cada vez más grandes, logrando con ello, acercarse a la probabilidad teórica del evento en cuestión, ahorrando tiempo. Por otro lado, esta forma de abordar el problema del concepto de probabilidad, permite dilucidar de si los casos favorables o totales están bien calculados, puesto que si es así, la simulación dará una buena aproximación, lo que haría revisar la solución hallada o bien confirmar que está correcta.

También, en la etapa de formulación, la simulación computacional ayudará al estudiante a atinarle a la solución del problema propuesta, logrando llegar al concepto que se desea establecer. La intuición puede fallar, y con la ayuda de la simulación del problema de Monty, pudimos darnos cuenta de que la probabilidad teórica del evento no era un $1 / 2$.

El enfoque frecuencial, debe constituirse en una etapa previa para un abordaje más formal en busca de la probabilidad teórica.

\section{Bibliografía}

[1] Antibí, A. Didáctica de las matemáticas, métodos de resolución de problemas. San José, Costa Rica: Serie CABÉCAR. 2000.

[2] Brousseau, G. Fundamentos y Métodos de la Didáctica de las Matemáticas. Traducción al castellano del artículo "Fondements et méthodes de la didactiques des mathématiques" publicado en la revista Recherches en Didactique des Mathématiques, 7(2):33-115, y realizada por Julia Centeno, Begoña Melendo y Jesús Murillo. 1986.

[3] Devore, J. Probabilidad y Estadística para Ingeniería y Ciencias. México: International Thompson Editores, $4 a$ ed. 1998.

[4] Gómez, M.. Elementos de estadística descriptiva. San José, Costa Rica: EUNED. 2000.

[5] Murillo, M. . Introducción a la matemática Discreta. Cartago, Costa Rica: Editorial Tecnológica de Costa Rica. 2004.

[6] Núñez, F. La enseñanza de la estadística en secundaria: Situación actual en Costa Rica, aproximación metodológica. En Facultad de Ciencias Físico Matemáticas, Benemérita Universidad Autónoma de Puebla. Memorias Primer Encuentro Nacional en la Enseñanza de la Probabilidad y la Estadística ( $1^{\circ}$ ENEPE), del 16 al 18 de junio del 2010. Puebla, México. 2010.

[7] Núñez, F. . Problemas de conteo: ¿Por qué son tan difíciles? Basado en las ideas de André Antibí. En Escuela de Matemática, Instituto Tecnológico de Costa Rica. Memorias IV Congreso Internacional de la Matemática Asistida por Computadora (4to CIEMAC), 1,2 y 3 de diciembre de 2005. Cartago, Costa Rica. 2005.

[8] Sanabria, G. Tópicos precedentes al estudio de la Teoría de Probabilidades. Cartago, Costa Rica: Publicaciones, ITCR. 2009.

[9] Sanabria, G. Una propuesta para la enseñanza de los Elementos de Análisis Combinatorio. En Facultad de Ciencias Físico Matemáticas, Benemérita Universidad Autónoma de Puebla. Memorias Primer Encuentro Nacional en la Enseñanza de la Probabilidad y la Estadística (1ENEPE), del 16 al 18 de junio del 2010. Puebla, México. 2010.

[10] Sanabria, G. \& Núñez, F. Una propuesta para introducir el estudio de las probabilidades: Probabilidad Frecuencial. En Facultad de Ciencias Naturales, Universidad Estatal a Distancia. Memorias III Encuentro de Enseñanza de la Matemática UNED, realizado en el INBio Parque, Heredia, Costa Rica, 3 y 4 de setiembre 2010. InBio Parque, Heredia, Costa Rica. 2010.

[11] Walpole, R, Myers, R, Myers, S. Probabilidad y estadística para ingenieros. USA: Prentice-Hall Hispanoamericana. S.A, Sexta Ed. 1999. 